AperTO - Archivio Istituzionale Open Access dell'Università di Torino

\title{
Fungal and Plant Tools for the Uptake of Nutrients in Arbuscular Mycorrhizas
}

\section{This is the author's manuscript}

Original Citation:

\section{Availability:}

This version is available http://hdl.handle.net/2318/1633108

since 2017-05-11T14:19:39Z

Publisher:

Elsevier

Published version:

DOI:10.1016/B978-0-12-804312-7.00007-3

Terms of use:

Open Access

Anyone can freely access the full text of works made available as "Open Access". Works made available under a Creative Commons license can be used according to the terms and conditions of said license. Use of all other works requires consent of the right holder (author or publisher) if not exempted from copyright protection by the applicable law. 
This is the author's final version of the contribution published as:

Giovannetti, M.; Volpe, V.; Salvioli, A.; Bonfante, P.. Fungal and Plant Tools for the Uptake of Nutrients in Arbuscular Mycorrhizas. Elsevier. 2017. pp: 107-128.

in

Mycorrhizal Mediation of Soil - Fertility, Structure, and Carbon Storage

The publisher's version is available at:

http://linkinghub.elsevier.com/retrieve/pii/B9780128043127000073

When citing, please refer to the published version.

Link to this full text:

http://hdl.handle.net/ 


\title{
CHAPTER 7
}

\section{Fungal and plant tools for the uptake of nutrients in Arbuscular mycorrhizas: a molecular view}

\author{
Marco Giovannetti ${ }^{1, \mathrm{a}}$, Veronica Volpe ${ }^{1, \mathrm{a}}$, Alessandra Salvioli ${ }^{1}$, Paola Bonfante ${ }^{1}$ \\ 1 Università degli Studi di Torino, Torino, Italy; 2 Gregor Mendel Institute (GMI), Vienna, Austria
}

\section{$\underline{\text { Introduction }}$}

One of the most ancient and successful strategies which plants adopted for nutrient acquisition is the formation of symbiotic association with Arbuscular Mycorrhizal (AM) fungi. The AM fungi can grow extensively in the soil thanks to an extended hyphal network and, therefore, reach unexplored soil niches, resulting in a high efficient mining for nutrients that are not accessible to the plant roots. On the other hand, the plant provides the fungus with up to $20 \%$ of its assimilated carbon, allowing the fungus to conclude its life cycle with the production of new spores. The AM symbiosis has been the default situation for plants since their appearance on Earth with the exception of those plants that lost this capacity during their evolution, such as Arabidopsis thaliana and plants belonging to the Brassicales order (Bravo et al., 2015).

The key structure of the nutrient exchange between the two symbiotic partners is a highly branched hypha, so-called arbuscule for its similarities to a tiny tree, completely surrounded by the invagination of the plant plasma membrane. The last is called perifungal membrane and it is enriched by plant nutrient transporters, which are induced by the AM fungal presence. Comparing fossils from 450 mya arbuscules (Remy et al., 1994) with confocal microscope pictures of the same structure from nowadays plants is sufficient to realize how well conserved have been the arbuscules, suggesting that mechanism controlling the fungal morphogenesis inside a plant cell have been maintained along the evolution, irrespectively of the plant identity (Bonfante and Genre, 2008). Despite the high level of conservation of the arbuscule across time, it is worth to remember that it constitutes an ephemeral structure lasting for around 5 days within a single plant cortical cell (Kobae and Hata, 2010). The mechanisms underlying the collapse of the arbuscule are still to be disentangled: a possible weekly checkpoint assures that the symbiosis is still efficient to the partners. It is known that, among different partners, a fine-tuned molecular mechanism regulates the symbiosis, while the exchange of nutrients is a major tool to assess it, as described by the biological market theory (Kiers et al., 2011). A detailed knowledge and research on nutrient uptake within AM symbiosis will have therefore two possible outputs: better understanding the impact of AM fungi on 
plant nutrition, improving their use in a more sustainable agriculture, and revealing which molecular drivers had led and controlled the establishment of this highly conserved and long-lasting relationship.

In this chapter, we will provide a detailed description of our current knowledge about fungal and plant molecular mechanisms involved in nutrient uptake within an Arbuscular Mycorrhizal symbiosis. In particular, three major macronutrients (nitrogen, phosphorous and sulfur) will be discussed with a special focus on old and new paradigms, future perspectives and open questions. The impact of AM symbiosis is not limited to changes in plant nutrient homeostasis at a local level but a whole set of plant responses happens in the green partner at a systemic level and has been highlighted in the last years, thanks to new available techniques.

\section{Nitrogen nutrition within Arbuscular Mycorrhizas}

Constituting up to $2 \%$ of plant dry weight, Nitrogen $(\mathrm{N})$ is above all required for aminoacids, nucleic acids and chlorophyll biosynthesis. It usually represents the limiting factor in plant growth and most of agricultural practices aim at rising $\mathrm{N}$ soil contents, through rotational crops and/or the massive use of N-based fertilizers. The high economic and environmental costs of $\mathrm{N}$ fertilizers (McSwiney \& Robertson 2005) are a great impulse towards the exploration of more sustainable practices: since $\mathrm{N}$ can be directly fixed from the atmosphere by some bacteria and Archaea, which possess the enzyme nitrogenase, and some plant species, as legumes, live in symbiosis with nitrogen-fixing bacteria, a challenging project is to engineering cereal crops with the capability to fix their own nitrogen (Rogers and Oldroyd, 2014). A more amenable approach is the understanding and exploitation of AM capacity of absorbing $\mathrm{N}$ from different substrates.

However, the actual contribution of AM fungi to plant $\mathrm{N}$ status is still under debate (Smith and Smith, 2011). As a first point, fungi -as plants and animals- need nitrogen not only for protein building, but also for assembling chitin, which is a a crucial component of their cell wall. We can therefore hypothesize that AM fungi uptake $\mathrm{N}$, as well as $\mathrm{Pi}$ and $\mathrm{S}$, from the soil for their own metabolism, and -only as a second step during the symbiosis functioning- they release the nutrients to their hosts.

In the past years AM symbiosis was associated with negative (George et al., 1995), neutral (Hawkins et al., 1999) and positive (Saia et al., 2014; Mensah et al., 2015) impacts on plant N status (for up-to-date review Correa et al., 2015; Bucking and Kafle 2015), depending on set-up conditions, and several authors postulated that the effect of AM symbiosis on plant $\mathrm{N}$ status is a merely consequences of improved P nutrition (Reynolds et al., 2005). It was also shown that the 
impact of AM symbiosis on plant $\mathrm{N}$ content and growth response was highly influenced by the AM fungal morphospecies used (Mensah et al., 2015). Indeed, it is clear that AM fungi are able to absorb different form of $\mathrm{N}$ and pass it to the plant in a similar way compared to what happen with $\mathrm{P}$ and other nutrients (Toussaint et al., 2004; Jin et al., 2005; Tanaka et al., 2005) and that the kinetics of $\mathrm{NH}_{4}{ }^{+}$acquisition by AM fungi show five times higher affinity compare to plants, therefore enabling to improve plant N nutrition even in soils with low N levels (Pèrez-Tienda et al., 2012).

In fact, the uptake of organic and inorganic N-sources from the soil via specialized transporters has been described for ectomycorrhizal (ECM), ericoid (Grelet et al., 2009) and AM fungi (Gobert and Plassard, 2008). It has been proposed that the release from fungus to the plant of these two different $\mathrm{N}$ forms depends on the nutritional and photosynthetic status of the plant (Chalot et al. 2006). The “traditional view” suggests an organic N-transfer under high C availability, whereas under C depletion the synthesis of organic $\mathrm{N}$ would be down regulated in the fungus, leading to the transfer of inorganic $\mathrm{N}$.

Inorganic $\mathrm{N}$ is present in the soil in two forms, as nitrate $\left(\mathrm{NO}_{3}{ }^{-}\right)$and ammonium $\left(\mathrm{NH}_{4}{ }^{+}\right)$, which are taken up from fungi. Studies on AM provide evidence that the $\mathrm{NH}_{4}{ }^{+}$is generally the preferential form absorbed by the fungal hyphae since it is considered energetically more efficient than $\mathrm{NO}_{3}{ }^{-}$ (Govindarajulu et al., 2005; Jin et al., 2005). However, the presence for a specific $\mathrm{N}$ form depends by the soil where the symbiosis occurs: $\mathrm{NO}_{3}{ }^{-}$is abundant in most agricultural soils, whereas $\mathrm{NH}_{4}{ }^{+}$ in many undisturbed or very acid soils (Bücking and Kafle, 2015). $\mathrm{NO}_{3}{ }^{-}$uptake is considered an active process and it is coupled to an $\mathrm{H}^{+}$-symport mechanism, while $\mathrm{NH}_{4}{ }^{+}$uptake involves an antiport mechanism with an $\mathrm{H}^{+}$efflux (Bago et al., 1996; Bago et al., 1997). Several $\mathrm{NH}_{4}{ }^{+}$and $\mathrm{NO}_{3}{ }^{-}$ transporters have been identified in AM fungi and they are localized both in extraradical or intraradical mycelium (Tisserant et al 2012). In Rhizophagus irregularis (previously Glomus intraradices) the expression of $\mathrm{NH}_{4}^{+}$-transporters GintAMT1 was higher in the extraradical mycelium and was induced by low level of $\mathrm{NH}_{4}{ }^{+}$(Lopez-Pedrosa et al., 2006). Moreover, it has been suggested that this transporter has a preferential role in the fungal $\mathrm{N}$ acquisition from the soil (Lopez-Pedrosa et al., 2006). By contrast, the other $\mathrm{NH}_{4}{ }^{+}$-transporters of $R$. irregularis, GintAMT2 was induced in the intraradical fungal structures indicating a preferential role in $\mathrm{N}$ acquisition during the symbiotic association (Pérez-Tienda et al., 2011). However, the localization of both GintAMT1 and GintAMT2 transporters via laser microdissection in the colonized cortical cells revealed that both transporters might have overlapping physiological functions in the symbiotic interface (Pérez-Tienda et al., 2011). A fungal $\mathrm{NO}_{3}{ }^{-}$transporter has been characterized in the extraradical mycelium of $R$. irregularis (Tian et al., 2010) and its expression was induced by the 
presence of $\mathrm{NO}_{3}^{-}$but it was suppressed by an increase of $\mathrm{NH}_{4}^{+}$or glutamine, a downstream metabolite, in the internal hyphae (Fellbaum et al., 2012).

AM fungi are also involved in the acquisition of organic N. These fungi release in the soil exoenzymes, as proteases, that convert the organic matter in N-containing compounds which are then absorbed by the fungi, thus providing a N-available form to the plants (Schimel and Bennett, 2004). Moreover, the AM fungi are able to directly absorb peptides, proteins and amino acids since they release peptidase and protease that cleave the $\mathrm{N}$ bound in this organic matter and free the nitrogenous monomers that are then absorbed by the fungi (Schimel and Bennett, 2004). Molecular investigations have reported that fungal amino acid transporters are expressed in the extraradical mycelium (Gachomo et al., 2009). The amino acid permease of Funnelliformis mosseae (previously Glomus mosseae), the GmosAAP1 was characterized and its expression was detected in the extraradical fungal structures and was induced by high level of organic N (Cappellazzo et al., 2008). The authors suggest that GmosAAP1 plays a role in the first steps of amino acid acquisition, allowing direct amino acid uptake from the soil and extending the molecular tools by which AM fungi exploit soil resources (Cappellazzo et al., 2008). Recently, Belmondo and co-authors have characterized the dipeptide transporter, RiPTR2, from the arbuscular mycorrhizal fungus $R$. irregularis. They reported that the RiPTR2 is expressed in the extraradical mycelium but also upregulated in the intraradical phase, suggesting a dual role of this transporter: in the uptake of organic $\mathrm{N}$ from soil and in the reabsorption of peptides from the interfacial apoplast (Belmondo et al., 2015).

The transfer of $\mathrm{N}$ from the soil to the plant via the fungus, follows the same mechanisms of other nutrients: the $\mathrm{N}$ uptake from soil by the fungal nutrient transporter into extraradical mycelium, the translocation along the hyphae and arbuscules and finally the uptake from the mycorrhizal interface by AM-inducible plant transporters in the root cortex (Smith and Smith 2011). When $\mathrm{NO}_{3}{ }^{-}$is taken up by AM fungi, it is reduced to nitrite by a nitrate reductase and then the nitrite is converted into $\mathrm{NH}_{4}{ }^{+}$by a nitrite reductase. An $\mathrm{NH}_{4}{ }^{+}$excess in the cells is considered to be toxic, thus the $\mathrm{NH}_{4}{ }^{+}$is rapidly assimilated into amino acids involving two pathways: the NAD(P)-glutamate dehydrogenase (GDH) or the glutamine synthetase-glutamate synthase (GS-GOGAT) pathway. This last pathway is that predominately used by AM fungi. The GS-GOGAT cycle was used to convert the absorbed inorganic $\mathrm{N}\left(\mathrm{NO}_{3}{ }^{-}\right.$and $\left.\mathrm{NH}_{4}{ }^{+}\right)$in arginine and then transported into the intraradical mycelium (Ngwene, et al. 2013). Before transfering the $\mathrm{N}$ source to the plant, the arginine is converted, through the urease cycle, in $\mathrm{NH}_{4}{ }^{+}$. Tian and co-authors demonstrated that the arginine in the mycorrhizal plants increased threefold and it is the most large amino acid owing to the fungal presence inside roots (Tian et al., 2010). 
During AM colonization an induction of many plant $\mathrm{N}$ transporters was observed. Among the 47 nutrient transporters which resulted to be AM-inducible in Lotus japomicus roots after 28 days of colonization (Guether et al., 2009), a high-affinity amino acid transporter, the LjLHT1.2 was identified (Guether et al., 2011). These transcripts were preferentially detected in arbusculated cells, suggesting that LjLHT1.2 transporter is involved in the re-uptake and in the recycle of amino acids from both mycorrhizal interface and cortical cells.

On the inorganic $\mathrm{N}$ side, several plant $\mathrm{NH}_{4}{ }^{+}$transporters have been identified in different species as L. japonicus, Sorghum bicolour and M. truncatula. In Lotus, the $\mathrm{NH}_{4}{ }^{+}$transporters LjAMT2;2 is reported as a high-affinity transporter and is preferentially expressed in arbusculated cells (Guether et al., 2009). Similar behaviour was shown also in Medicago (Gomez et al., 2009), in soybean (Kobae et al., 2010) and in sorghum, where the expression of SbAMT3;1 and SbAMT4 was induced only in arbusculated cells (Koegel et al., 2013). As what was shown with other nutrient transporters, also the expression of ammonium transporters in rice are influenced by different factors: a group of these genes is overexpressed at lower N levels, such as OsAMT1;1, OsAMT1;3 and OsAMT3;3, whereas some other transporters are exclusively influenced by the fungal presence, such as OsAMT3;1 (Fiorilli et al., 2015), well mirrored by glutamate synthase expression (PèrezTienda et al., 2014).

Recently, it has been demonstrated that the AMT2;3 of Medicago is involved in the suppression of premature degeneration of arbuscules in the mtpt4 mutants grown under $\mathrm{N}$ stress, evidencing therefore a crosstalk between $\mathrm{N}$ and $\mathrm{P}$ metabolism in AM roots and a key role for the ammonium transporter in regulating a functional arbuscule formation. The functional role in $\mathrm{NH}_{4}{ }^{+}$transporter was reported only for AMT2;4 of Medicago, suggesting that AMT2;3 could be more involved in Nsensing/signalling pathway (Breuillin-Sessoms et al., 2015). A further confirmation of this interconnection is that a condition of low $\mathrm{P}$ and low $\mathrm{N}$ in the soil can dramatically increase the percentage of AM colonization (Bonneau et al., 2013) and, interestingly, in Petunia the nitrogen starvation is partially overruling the suppressive effect of high P nutrition on arbuscular formation. (Nouri et al., 2015).

There is, therefore, strong evidence that AM fungi are able to acquire $\mathrm{N}$ from organic and inorganic sources and transfer it to the plant; $\mathrm{N}$ itself is equally contributing to the fine-tuned regulation of AM establishment and functioning acting together with the phosphate, even thought the molecular mechanisms of the nutrient interplay are still largely unknown.

\section{Phosphate transport in the arbuscular mycorrhizal symbiosis}


Phosphorous (P) is an essential macronutrient for the proper growth and functioning of plants. The predominant form of $\mathrm{P}$ in the soil is the dihydrogen phosphate ion $\left(\mathrm{H}_{2} \mathrm{PO}_{4}{ }^{-}, \mathrm{Pi}\right)$ (Nussaume et al., 2011). This anion in the soil is scarcely mobile and strongly interacts with iron, aluminium and calcium, limiting its availability for the plants and leading the Pi concentrations at very low levels $(<10 \mu \mathrm{M})$ in the root-soil interface (Smith et al., 2011). Moreover, another factor that restricts the $\mathrm{Pi}$ availability in the soil is its assimilation by microbes. To overcome scarce levels of Pi, plants have evolved a range of strategies to increase both Pi uptake and Pi availability in the soil (Marschner, 1995) and the AM symbiosis represents the long-lasting and more efficient tool to address this function. In fact AM fungi can uptake Pi in a highly efficient way convert it in polyphosphate molecules for movement through the mycelia. The polyphosphates are then hydrolyzed, probably by the phosphatase activities, again in Pi for the transport from the fungal structures, as intercellular hyphae or arbuscules, into root cortical cells. It has been demonstrated that the fungal contribution in the plant Pi acquisition ranges from a small percentage to almost all the acquired Pi (Smith et al., 2003, 2004). In this way, AM fungi provide a very effective and indirect pathway, called AM pathway, which interacts with the direct pathway, where root epidermal cells and root hairs are involved in the Pi uptake from the soil (Smith et al., 2011). The interplay between the direct and indirect pathway for Pi uptake was recently studied at molecular levels (Watts-Williams et al. 2015). The authors investigated the distal and local effects of AM colonization on the direct root Pi uptake, suggesting that AM fungi reduce the direct root Pi uptake activity locally but not in a distal, non-colonized patch of root (Watts-Williams et al. 2015).

Some fungal Pi transporters appear to be responsible for the first step of this symbiotic Pi transport and three AM Pi transporters were identified so far: GmosPT from Glomus mosseae, GvPT from Glomus versiforme, GiPT from Glomus intraradices, as well as one from Gigaspora margarita, on the basis of a transcriptomic data set (Salvioli et al, 2016). They are all expressed in the extraradical hyphae and GmosPT showed also an expression in the intraradical hyphae (Javot et al., 2007).

AM fungi are able to efficiently absorb Pi from the soil and accumulate it as PolyP. The rapid and massive accumulation of polyP in fungal mycelia is accompanied by near-synchronous and nearequivalent uptake of $\mathrm{Na}^{+}, \mathrm{K}+, \mathrm{Ca} 2+$ and $\mathrm{Mg} 2+$, with a parallel regulation of gene expression responsible for mineral uptake, $\mathrm{Pi}$ and nitrogen metabolism, and maintenance of cellular homeostasis (Kikuchi et al., 2014).

The release of Pi within the root cortex is accompanied by a significant upregulation of plant $\mathrm{Pi}$ transporters which have been identified in the following species: Medicago (Harrison et al., 2002; Javot et al., 2007), rice (Paszkowski et al., 2002), Lotus (Guether et al., 2009; Volpe et al., 2015), Astragalus sinicus (Xie et al., 2013), potato (Nagy et al., 2005), maize (Nagy et al., 2006), various 
cereals (Glassop et al., 2005), tomato (Nagy et al. 2005; Balestrini et al., 2007; Xu et al., 2007), poplar (Loth-Pereda et al., 2011) sorghum and flax (Walder et al., 2015). These transporters belong to the PHT1 family of Pi transporters and they show an amino acid sequence conserved from fungi to plants. They are 500-600 amino acids long and they share a common topology with 12 predicted membrane-spanning domains of 17-25 amino acids residues that are arranged in a helix. The membrane-spanning regions are separated in two groups of six domains by a hydrophilic and charged loop (Nussaume et al., 2011; Johri et al., 2015). Furthermore, these transporters are specific to AM symbiosis since they are expressed strictly in response to AM fungi and they are localized in the perifungal membrane, specifically defined as periarbuscular membrane (PAM), the site where the nutrient exchange happens between the two symbionts (Harrison et al., 2002). One of the most characterized mycorrhiza-induced Pht1 genes was MtPT4 of Medicago truncatula (Harrison et al., 2002). The MtPT4 protein immunolocalization revealed the signal around the fine arbuscular branches but not in the regions around the hyphal trunk. Moreover, MtPT4 was detected only in developing and mature arbuscules (Harrison et al., 2002; Javot et al., 2007). The heterologous expression of MtPT4 in yeast revealed a lower affinity for Pi, with an apparent $\mathrm{Km}$ between 493 and $668 \mu \mathrm{M}$ (Harrison et al., 2002). The translocation of phosphate from the periarbuscular space throughout the periarbuscular membrane requires an $\mathrm{H}+$-energy gradient that is provided by an $\mathrm{H}^{+}$ATPases (Krajinski et al., 2014; Wang et al., 2014a).

During the AM colonization, the Pi transporter genes that are involved in the direct pathway can be down-regulated, independently of Pi status. MtPT1, MtPT2 and MtPT3 of M. truncatula are well characterized (Bucher et al., 2001; Liu et al., 2008) and they belong to PHT1 family, but differently from MtPT4 their mRNA level were reduced in mycorrhizal roots, excluding their involvement in Pi transfer at the arbuscular interface (Chiou et al., 2001). In M. truncatula two other PHT1 genes of the direct pathway have been identified: MtPT5 and MtPT6, both genes are expressed in nocolonized root but they are also affected in AM plants (Grunwald et al., 2009). The expression of the direct $\mathrm{Pi}$ transporter genes in non-mycorrhizal plants is regulated by $\mathrm{Pi}$ starvation signaling pathway. In Arabidopsis, which is not colonized by AM fungi, a complex network controls the regulation of gene involved in the direct pathway, where the transcription factor PHR1 is involved. PHR1 bind to the cis-regulatory P1BS motif that is present in the promoter region of most PHR1dependent Pi starvation-induced gene (Rubio et al. 2001). Components of this network are conserved in plants. Recently, it has been investigated the molecular mechanisms that lead to the transcriptional activation of PHT1 genes in response to AM symbiosis (Chen et al., 2010). Two conserved motifs were identified into AM-induced PT promoters, one of this is P1BS and the other one is named MYCS (mycorrhiza transcription factor binding sequence) and both are involved in 
the activation of AM-responsive PT genes (Chen et al., 2010). In Arabidopsis plants, four WRKY proteins have been reported to be involved in Pi starvation signaling pathway (Devaiah et al., 2007; Chen et al., 2009; Wang et al., 2014b; Su et al., 2015). WRKY42, WRKY45 and WRKY75 have been identified as positive regulators of PHT1;1 genes (Devaiah et al., 2007; Su et al., 2015).

Phosphate levels and phosphate transporters are crucial regulators of AM symbiosis. When plants are grown at high level of phosphate, AM colonization is drastically reduced or completely impaired, depending on the plant and on the phosphate concentration. (Balzergue et al. 2011; Breuillin et al. 2010). Moreover, when AM-inducible transporters are non-functional or downregulated by RNAi, the formation of arbuscule is impaired. (Harrison et al., 2002; Paszkowski et al., 2002; Xie et al., 2013; Volpe et al., 2015) and the transfer of the phosphate ion itself across the periarbuscular membrane has been proposed to act as a cell signal triggering the accomplishment of the AM symbiosis (Yang et al., 2012). However the suppressive effect of high $\mathrm{Pi}$ on root colonization by AMF and also the degeneration of arbuscules in mtpt4 mutants is partially overruled by nitrogen (N) starvation (Nouri et al., 2014; Breuillin-Sessoms et al. 2015). It appears that a complex interplay between nutrient homeostasis, nutrient acquisition and nutrient sensing is able to regulate the AM colonization but its mechanism needs still to be elucidated.

Recently, Volpe et al. (2015) demonstrated that the expression of MtPT4 gene and another AMinduced PT gene, LjPT4 from Lotus japonicus, was also found in the root tips of non-colonized plants, suggesting that both genes could act upstream of the Pi-sensing cellular machinery, as potential membrane Pi transceptors (Box 1). The authors suggested that both AM-responsive phosphate transporters play a role in root tips, creating a link among Pi-perception, root branching and Pi-signalling mechanism. A similar role for phosphate sensing was also proposed for OsPT13, a rice AM-responsive gene that show no function in phosphate transfer but is necessary for the correct formation of the arbuscule. (Yang et al., 2012).

To date, the concept of transceptor has been described only in other two plants, Arabidopsis thaliana and L. japonicus, where the nitrate transporter AtNRT1.1 and the ammonium transporter LjAMT1;3, respectively, have been proposed to act as sensor (Remans et al., 2006; Rogato et al., 2010). AtNRT1.1 is involved in the regulation of $\mathrm{NO}_{3}{ }^{-}$-responsive genes and in root growth and development, activating $\mathrm{NO}_{3}{ }^{-}$-sensing/signalling pathway. Taken together, these works are opening an interesting scenario where the multiple functions of these nutrient transporters are highlighted and could represent an important link between plant root development, nutrient sensing and AM formation. 


\section{$\underline{\text { Sulfur metabolism and AM symbiosis }}$}

Together with phosphorous, nitrogen, potassium, calcium and magnesium, sulfur ( $\mathrm{S}$ ) is an essential macronutrient for plants. Within a plant cell, it is needed as a backbone for cell wall and cell membrane formation, being a necessary part of sulfolipids, but also as a main component of essential aminoacids, such as cysteine and methionine (see Takahashi et al., 2011 for a review). Glutathione, an important S-containing redox controller, is a key metabolite in a plethora of plant cell developmental processes (Foyer et al., 2009). Beside basic metabolism, S plays an important role in plant secondary processes, and in Arabidopsis it is present in more than 400 metabolites (Gläser et al., 2014). Even though the majority of the functions of these metabolites are still unknown, some have been the focus of many studies thanks to their pivotal action in plant immunity (i.e. camalexin, Nawrath and Métraux, 1999) or flavor quality (i.e. organosulfur compounds from Allium species, Kyung and Lee, 2001).

Across $19^{\text {th }}$ century, $\mathrm{S}$ used to be abundant in the soil, not limiting plant growth, because of the sulfide and S dioxide gasses coming from heavy industries, oxidized to sulfate in the atmosphere and recycled to biosphere as rain falls (Lehmann et al., 2008). But starting from last decades, S limitation is becoming an issue for many farmers, with a dramatic effect on final products: bread leavening relies on the presence in wheat flour of high concentration of proteins, highly correlated with soil sulfur levels (Granvogl et al., 2007). When plants are deprived on S, they show strong phenotypes such as yellowing of the leaves, reduced growth and seed number. Furthermore, during the sulfate deficiency the biosynthesis of hormone, such as auxin and jasmonate, is induced and sulfate uptake and assimilation are regulated by ethylene, jasmonate, abscisic acid and other phytohormones (Koprivova and Kopriva, 2016).

Notwithstanding the major role that $\mathrm{S}$ plays in plant health and metabolism, the mycorrhizal community has started relatively recently to address the issue, investigating what is the impact of mycorrhization on plant S nutrition and which are the genetic determinants for mycorrhizal sulfur uptake.

Beside preliminary and pioneering experiments comparing mycorrhizal and non-mycorrhizal plant capacity of uptaking ${ }^{35} \mathrm{~S}$ from the soil 8-cm distant from the root (Rhodes and Gerdemann, 1978), the demonstration that AM fungi are able to absorb both organic and inorganic $S$ forms and transfer them to the plant root was elegantly given by Allen and Shachar-Hill (2009) with the use of transformed carrot roots and Rhizophagus irregularis cultures and, later, confirmed in a twocompartment system with entire Medicago plants (Sieh et al., 2014). 
However, the comparison between sulfate content in mycorrhizal and non-mycorrhizal plants allowed to show how the presence of the fungal symbiont can positively improve plant sulfate content especially, but exclusively, when plants are grown in low-sulfate conditions. This effect was shown on Medicago truncatula (Casieri et al., 2012; Sieh et al., 2014), Lotus japonicus (Giovannetti et al., 2014), maize (Gerlach et al., 2015) both in semi-controlled environment and in field experiments.

In order to investigate which genes could be responsible of sulfate transfer with AM symbiosis, different transcriptomic approaches have been adopted, both target and non-target. Throughout qRT-PCR, the transcription of putative sulfate transporters in Medicago was analyzed during AM interaction, both at high and low level of S, showing the regulation induced by the fungal presence of many different transporters (Casieri et al., 2012; Sieh et al., 2014), even though not always consistent between the different studies. The intricacy of these kinds of studies is the discrimination between direct effects caused by the fungal presence or by indirect effects due the modified nutrient status in mycorrhizal roots. Moreover, in the work of Sieh et al. (2014) it has been reported that the efficiency of S uptake in plant root was dependent from Pi availability, observing an increase of S transfer when the soil Pi content was low.

In the last years, the use of microarray and RNAseq allowed a deeper characterization of key candidate genes in plant and fungal transcriptome, potentially involved in arbuscular mycorrhizal S uptake. In L. japonicus, a strong regulation of a sulfate transporter (SULTRs) in roots colonized either by Gigaspora margarita or $R$. irregularis was shown by two different experiments (Guether et al., 2009; Handa et al., 2015) and a consequent molecular characterization demonstrated its double role in mediating both direct sulfate uptake from soil, when plants are in a S-starvation condition, and a subsequent activation in arbuscule-containing cells, once the symbiosis is formed. LjSultr1;2 is not the only sulfate transporter regulated during AM symbiosis. In fact plants possess dozens of sulfate transporters and a fine-tuned regulation allows balancing between storage, catabolism and root-to-shoot movement of sulfate pool.

Sulfate transporters can be distinguished in four functional groups, based on their sequences and their different activation (for a review see Gigolashvili et al., 2015). Mainly from Arabidopsis, we learned that Group 1 encodes high affinity SULTRs and are usually mediating direct uptake of sulfate from the soil, group 2 are low affinity transporters and responsible for long distance translocation of sulfate (Takahashi et al., 2000), group 3 is still largely unknown and diverse and group 4 encodes vacuolar sulfate exporters (Buchner et al., 2004; Takahashi et al., 2011) and usually regulating sulfate homeostasis. The structures and transcriptional regulation of SULTRs 
were also characterized in other plant species, such as Brassica oleracea, poplar, wheat, and $M$. truncatula (Buchner et al., 2004, 2010; Durr et al., 2010).

A recent microarray experiment was specifically focused on Medicago root and leaf transcriptome responses to S-deficiency in plants interacting with the AM fungus $R$. irregularis: several hundreds genes resulted to be regulated both by the fungal presence and by the $\mathrm{S}$ status. In root tissues, low $\mathrm{S}$ treatment induced the downregulation of several genes, among which genes coding for thiosulfate and glutathione sulfurtransferases, gibberellin oxidases and quinone oxidoreductases. Interestingly, this effect was less evident in mycorrhizal plants compared to non mycorrhizal plants (Wipf et al., 2014). The way to understand which genes are regulating sulfur metabolism in plants is still long but a further characterization of candidate genes coming from -omics studies will better elucidates this complex system.

Interestingly, both in yeast (Kankipati et al., 2015) and in the model plant Arabidopsis thaliana, sulfate transporters have been proposed to act not only in transferring sulfate through cell membranes but also in sulfate sensing, as shown by their capacity to regulate gene transcription of sulfate metabolism, independently on sulfate transfer (Zhang et al., 2014). A parallel role in arbuscular mycorrhizal symbiosis it is worth to be investigated, since nutrient exchange is a key regulator of an established symbiosis and, so far, exclusively phosphate transporters have been demonstrated to play such a role in the formation of a functional arbuscule (Harrison et al., 2002).

\section{4: From root to shoot and back: evidence for a systemic signalling and gene regulation in mycorrhizal plant}

Notwithstanding the efforts spent to understand the molecular basis of arbuscular mycorrhiza establishment and nutrient exchange dynamics, little is known about the systemic spread of the mycorrhizal signal. The AM symbiosis in fact entails in the plant host a plethora of effects including improved growth and biomass production, tolerance to both biotic and abiotic stresses, changes in metabolite production (van der Heijden et al., 2006; Ruiz-Lozano et al., 1996, Smith and Smith 2008, Pozo and Azcón-Aguilar 2007), and the idea that such diverse systemic outcomes could not be uniquely attributed to the improved nutritional status is largely acknowledged.

In the last years many authors addressed the question whether the mycorrhiza establishment in the root compartment may entail a long-distance molecular impact, and the interest was first of all directed towards the core of the mycorrhiza functioning, namely the nutrient exchange. If it is well acknowledged that the expression of nutrient transporter genes (mainly $\mathrm{P}$ and $\mathrm{N}$ ) is regulated in mycorrhizal roots, whether the regulation is systemic or not remained an open question. Using the split-root system, that allows to simultaneously obtain a mycorrhizal and a non mycorrhizal root 
compartment from the same experimental plant, different studies approached this question. In 2013 Koegel and colleagues analyzed the expression of several transporters in Sorghum bicolor and found that two specific ammonium transporters were locally but not systemically induced by mycorrhization, while a strict AM-inducible Pi transporter was indeed overexpressed in non mycorrhizal roots from myc plants (Koegel at al., 2013). This patchy situation has been confirmed by other studies. A recent research on wheat revealed that root colonization by AMF locally but not systemically up-regulated the expression of AM-inducible Pi transporters, while distal effects on the expression of nitrate and ammonium transporter genes varied depending on the specific gene and AM species considered (Duan et al., 2015). On the same line, another study indicated that colonization by AM fungi decreased expression of direct $\mathrm{P}$ transporter genes (that mediate mycorrhizal-independent P uptake) locally, but not systemically, and direct root P uptake was only weakly influenced on distal root portions (Watts-Williams et al., 2015).

On the whole, the results obtained suggest that the colonization by AMF produces a signal that could be spread to the non-colonized roots, although this phenomenon does not seem to represent a major regulatory pathway in mycorrhizal symbiosis.

Taking a step back in the symbiosis progression, it is well known that a systemic bi-directional signaling is involved in regulating the establishment of arbuscular mycorrhiza.

For example, the root colonization by an AM fungus is able to negatively regulate further mycorrhization, and this phenomenon is known as autoregulation (Staehelin et al., 2011). Taking advantage from the split-root system, many studies proved that initial mycorrhization of one root half is able to suppress AMF colonization on the other half (Vierheilig et al., 2000; Catford et al., 2003). In analogy with what already well described for nodulation, the signaling molecules involved in the mycorrhizal autoregulation are proposed to migrate in the xylem and in the phloem to transfer the signal from the root to the shoot and then back to the belowground compartment (Staehelin et al 2011). Furthermore, other researches demonstrate that the suppression of mycorrhization observed under high phosphate availability is mainly dependent on the shoot $\mathrm{P}$ levels rather than the $\mathrm{P}$ status experienced by the roots, thus implying the involvement of a shootderived signal (Breuillin et al., 2010; Balzergue et al., 2011).

These data prove that not only the suppressive signal works systemically, but such a feedback also requires the activity of signaling and sensing components localized in plant districts other than root.

In the examples so far described, the molecular signaling was involved in events that are instrumental in the symbiosis itself. But does it also play a role in inducing the long distance effects 
that feature the mycorrhizal outcome on the whole plant? Emerging evidence supports this view as in the case of systemic resistance to disease.

The ability of AMF to protect plants from pathogen attack through induction of systemic resistance is long time recognized (Cordier et al., 1998). Interestingly, nutrient supply experiments have revealed that such effect is not a consequence of the improved nutritional status (Fritz et al., 2006). In early 2000, Maria Pozo and colleagues studied at the biochemical level the defense response of tomato against Phytophtora parasitica in mycorrhizal and non mycorrhizal roots. They found that AMF inoculation reduced disease symptoms, and systemic alteration of the activity of defenserelated enzymes was also observed in non-mycorrhizal roots of mycorrhizal plants (Pozo et al., 2002). This “mycorrhiza-induced resistance” acts as a systemic priming of defense against a wide range of attackers, and its spread seems to involve a complex hormonal signaling in which jasmonic acid and salicilic acid play a pivotal role (Cameron et al., 2013). In a very recent work, mycorrhizal tomato plants challenged with the fungal pathogen Alternaria solani responded to the attack with a strong induction of pathogenesis-related genes, as well as other defense-related genes in leaves. However, pathogen infection did not induce these genes and enzymes in a jasmonate-biosynthesis defective plant, indicating that the JA signaling is an essential component of the priming mechanism triggered by mycorrhiza (Song et al., 2015). At the same time these data also confirm that the long-distance protective effect involves the modulation of transcription of defense-related genes in plant districts far from the root. The same class of defence-related genes is also triggered when plants are challenged with arbuscular mycorrhizal spore exudates or the purified molecules contained in those exudates, such as short chitin oligomers (Giovannetti et al., 2015).

In this respect, a pioneer research was performed by the Maria Harrison group, who employed a microarray and q-PCR based strategy to explore local and systemic transcriptional responses to AMF in Medicago truncatula roots and shoots (Liu et al., 2007). They observed a specific transcriptome remodeling in shoots as a consequence of root colonization by AMF, and in particular genes dealing with stress and defense responses were up-regulated. Accordingly, M. truncatula plants showed increased resistance to a virulent bacterial pathogen. Another point of novelty of this work is that the experiment was designed to avoid a significant enhancement of plant phosphate nutrition by the AM fungus. The fact that, under these conditions, important transcriptome changes were still recorded in mycorrhizal plants strengthens the idea that alterations in gene expression are not necessary linked to the improvement of phosphate nutrition.

After that, many other works analyzed changes in gene expression induced systemically by AM symbiosis, often employing microarray and the more recent deep sequencing approach (Fiorilli et 
al., 2009; Salvioli et al., 2012; Zouari et al., 2014; Cervantes-Gámez et al., 2015). It is interesting to notice that in all the papers cited the studied plant species was tomato; indeed, in the last decade much attention has been paid to dissect the mechanisms that feature the mycorrhizal status in crop plants, with a particular focus on agronomically relevant traits. Even if the improvement of such traits could be more easily put in relation with the enhanced nutrition and with the generically defined "growth effect" triggered by AMF, the advent of more refined molecular techniques enabled researchers to reveal the fine shaping of the plant physiology underneath this systemic effect, that implies deep molecular and metabolic rearrangement in distal parts of the plant. These investigations clearly indicate that mycorrhiza establishment can improve the nutritional value of different crops. Following a biochemical approach, Baslam and colleagues demonstrated that enhanced levels of carotenoids, chlorophylls and tocopherols are induced in leaves from mycorrhizal lettuces (Baslam et al., 2013). On the same line, mycorrhization improved the nutrient quality of tomato fruits, since lycopene, carotenoid and volatile compound contents were significantly increased (Giovannetti et al., 2012; Hart et al., 2015). In recent works from our group, changes in gene expression in tomato fruit were accompanied by phenological modifications as an accelerated flowering and fruiting time, an overall increase in fruit yield as well as qualitative and quantitative changes in aminoacid profile (Salvioli et al., 2012). Interestingly, when the experiment was designed to avoid nutrient starvation in control plants, phosphate and sulphate transporters turned up to be up-regulated in fruits from myc plants, suggesting that this regulation is mycorrhizal-specific and not only related to the improved nutritional status (Zouari et al., 2014).

Although interesting, these studies often suffer the same weakness represented by the difficulty in clearly connecting among them the results obtained at different layers of the analysis, and in scaling up from the molecular to the phenotypical level the results obtained. An integrated approach might be more effective in dissecting the complexity of the whole system. A recent attempt to do so was peformed by Gerlach and colleagues, who dissected systemic responses in maize to AM symbiosis at the single-leaf level, applying a multi-profiling analysis that included comparative ionomic, transcriptomic and metabolomic, as well as phenotypical analyses. With this strategy the Authors could link the observed growth enhancement with robust changes in elemental leaf composition, an increase in anthocyanin and lipid metabolism, a reprogramming of leaf $\mathrm{C} / \mathrm{N}$ ratio and the induction of defence-related genes (Gerlach et al., 2015).

The evidence provided thus points to the involvement of long distance "mycorrhizal signals" flowing from root to shoot and back. To date, the major candidates for this role are hormones and miRNAs. The involvement of phytohormones in the establishment and functionality of the AM 
symbiosis is already well acknowledged, and a consistent bulk of data also supports their role in the spread of mycorrhiza-induced resistance (Cameron et al., 2013). In addition, there is fresh evidence suggesting that micro-RNAs (miRNAs) also play a regulatory role during AM symbiosis (Devers et al., 2011). MiRNAs are 20-25-nucleotide long phloem-mobile RNAs, that can regulate the plant physiology in response to diverse external stimuli, including nutrient availability (Kulcheski et al., 2015). Interestingly, an in silico analysis on solanaceous genomes identified Pi starvation and arbuscular mycorrhiza related cis elements in the putative promoter regions of some miRNA genes (Gu et al., 2015), strengthening the hypothesis of a role for miRNA in mediating the plant response to mycorrhization.

In conclusion, the well acknowledged growth effect exerted by AMF is accompanied by molecular changes that imply a mycorrhiza-specific signalling and a fine-tuning of the metabolism of distal part of the plant host. Such systemic outcome is not easy to be studied as it is influenced by many factors (i.e. the specific plant-fungus couple, the surrounding environment, the interaction with other microbes...), but the fine understanding of how AMFs shape the plant physiology represents a frontier towards the exploiting of mycorrhizal symbiosis in sustainable agriculture.

\section{Conclusions and perspectives}

Notwithstanding their biotrophic nature, AM fungi possess amazing capacities to extract mineral nutrients from the soils and genome sequencing (Tisserant et al., 2013) clearly demonstrated that their enzyme repertoire is rich in such transporters, differently from the pathogenic biotrophs which live on leaves and are fully dependent on their host also for the nutrient uptake and not only for carbon.

In this context AM fungi release a part of these nutrients to the plants inducing the expression of many plant transporters and activating the so called symbiotic pathway. However, how much nutrients does the fungus retain for its own metabolism and how much is transferred to the plant is still unclear, as well as the real environmental conditions which control these exchanges. Studies based on the transcriptional profile of arbusculated cells collected with a laser dissector (Balestrini et al 2007; Fiorilli et al.) strongly suggest that the fungal control between the released and the reuptaken nutrients operated a the arbuscule level. The understanding of these issues could be crucial for a better exploitation of AM symbiosis and modern techniques, such as NanoSIMS, (mettere ref) will help to reveals such nutrient traffics in vivo.

Arbuscular mycorrhizal inducible plant transporters are well characterized but new functionalities have been recently unveiled, such as nutrient sensing, even in the absence of the fungal partner, 
opening the question of their role in an evolutionary context. During the land conquest, plants had to cope with a strong mineral nutrient deprivation and roots were not present yet (Bonfante \& Genre, 2008), therefore nutrient sensing could have represented a driving force in different nutrient uptake strategies (Volpe et al., 2015). This function may also exert a control on the root architecture creating a strict link between plant development, environmental conditions and AM establishment. Deciphering this network could help in understanding factors which limit AM colonization in agricultural ecosytems.

Nevertheless, in order to increase plant productivity and agriculture sustainability, there's a need to fully understand and domesticate AM effects at a systemic level with open field studies and multidisciplinary approach. One of the open question is the reciprocal impact of plant photosynthesis and AM fungi: on one hand they act as C sinks, but on the other they can stimulate plant photsyntesis.

Finally, it is more and more clear that each ecotype evolved a specific adaptation to local environments and, as a consequence, specific genetic alleles regulated plant developmental process in a peculiar ways. Genome Wide Association Studies are a key tool that guarantees the identification of natural alleles and their effect on plant phenotype (Gentzbittel et al., 2015). In this framework, it will be interesting understanding if those differences among ecotypes are also influenced by the presence of AM symbiosis, as shown for example in maize (Sawers et al., 2016).

In this context, as shown for example in maize (Sawers et al., 2016) where... it will be interesting understanding where the differences among ecotypes revealed by by GWAS are influenced by the presence of AM symbiosis. This will be the key to decipher the systemic responses

\section{References}

Balestrini, R., Gomez-Ariza, J., Lanfranco, L., and Bonfante, P.(2007). Laser microdissection reveals that transcripts for five plant and one fungal phosphate transporter genes are contemporaneously present in arbusculated cells. Mol.Plant MicrobeInteract. 20, 1055-1062.

Balzergue C., Puech-Pagès V., Bécard G. \& Rochange S.F. (2011) The regulation of arbuscular mycorrhizal symbiosis by phosphate in pea involves early and systemic signalling events. Journal of Experimental Botany 62, 1049-1060. 
Breuillin F., Schramm J., Hajirezaei M., Ahkami A., Favre P., Druege U., .., Reinhardt D. (2010) Phosphate systemically inhibits development of arbuscular mycorrhiza in Petunia hybrida and represses genes involved in mycorrhizal functioning. The Plant Journal 64, 1002-1017

Breuillin-Sessoms F., Floss D.S., Gomez S.K., Pumplin N., Ding Y., Levesque-Tremblay V., ..., Harrison M.J. (2015) Suppression of arbuscule degeneration in Medicago truncatula phosphate transporter4 mutants is dependent on the ammonium transporter 2 family protein AMT2;3. The Plant Cell 27, 1352-1366

Bucher M., Rausch C., Daram P. (2001). Molecular and biochemical mechanisms of phosphorus uptake into plants. Journal of Plant Nutrition and Soil Science 164, 209-217.

Chen Y.F., Li L.Q., Xu Q., Kong Y.H., Wang H., and Wu W.H. (2009). The WRKY6 transcription factor modulates PHOSPHATE1 expression in response to low Pi stress in Arabidopsis. Plant Cell 21, 3554-3566.

Chen A, Gu M, Sun S, Zhu L, Hong S, Xu G (2011). Identification of two conserved cis-acting elements, MYCS and P1BS, involved in the regulation of mycorrhiza activated phosphate transporters in eudicot species. New Phytol 189, 1157-69

Chiou T.J., Liu H., Harrison M.J. 2001. The spatial expression patterns of a phosphate transporter (MtPT1) from Medicago truncatula indicate a role in phosphate transport at the root/soil interface. The Plant Journal 25, 281-293.

Devaiah B.N., Karthikeyan A.S., and Raghothama K.G. (2007). WRKY75 transcription factor is a modulator of phosphate acquisition and root development in Arabidopsis. Plant Physiol 143, 17891801.

Glassop, D.,Smith, S.E., and Smith, F.W.(2005).Cereal phosphate transporters associated with the mycorrhizal pathway of phosphate uptake into roots. Planta 222, 688-698. 
Grelet GA, Meharg AA, Duff EI, Anderson IC, Alexander IJ. Small genetic differences between ericoid mycorrhizal fungi affect nitrogen uptake by Vaccinium. New Phytol. 2009;181(3):708-18. doi: 10.1111/j.1469-8137.2008.02678.x. Epub 2008 Nov 13.

Grunwald U., Guo W., Fischer K., Isayenkov S., Ludwig-Müller J., Hause B., Yan X., Küster H., Franken P. 2009. Overlapping expression patterns and differential transcript levels of phosphate transporter genes in arbuscular mycorrhizal, Pi-fertilised and phytohormone-treated Medicago truncatula roots. Planta 229, 1023-1034.

Guether M., Balestrini R., Hannah M., He J., Udvardi M.K. \& Bonfante P. (2009) Genome-wide reprogramming of regulatory networks, transport, cell wall and membrane biogenesis during arbuscular mycorrhizal symbiosis in Lotus japonicus. New Phytologist 182, 200-212

Harrison M.J., Dewbre G.R. \& Liu J. (2002) A phosphate transporter from Medicago truncatula involved in the acquisition of phosphate released by arbuscular mycorrhizal fungi. The Plant Cell 14, 2413-2429

Javot H., Penmetsa R.V., Terzaghi N., Cook D.R. \& Harrison M.J. (2007) A Medicago truncatula phosphate transporter indispensable for the arbuscular mycorrhizal symbiosis. Proceedings of the National Academy of Sciences, USA 104, 1720-1725

Johri A.K., Oelmüller R., Dua M., Yadav V., Kumar M., Tuteja N., Varma A., Bonfante P., Persson B.L. and Stroud R. M. (2015) Fungal association and utilization of phosphate by plants: success, limitations and future prospects. Frontiers in Microbiology 6, 984-997.

Krajinski F., Courty P.E., Sieh D., Franken P., Zhang H., Bucher M., ..., Hause B. (2014) The H+ATPase HA1 of Medicago truncatula is essential for phosphate transport and plant growth during arbuscular mycorrhizal symbiosis. The Plant Cell 26, 1808-1817

Liu J., Versaw W.K, Pumplin N., Gomez S.K., Blaylock L.A., Harrison M.J. (2008). Closely related members of the Medicago truncatula PHT1 phosphate transporter gene family encode phosphate transporters with distinct biochemical activities. Journal of Biological Chemistry 283, 24673-24681. 
Loth-Pereda,V., Orsini E.,Courty, P.E., Lota, F. , Kohler, A., Diss,L., Blaudez, D., Chalot,M., Nehls,U., Bucher,M., and Martin,F.(2011). Structure and expression profile of the phosphate Pht1 transporter gene family in mycorrhizal Populus trichocarpa. Plant Physiol. 156, 2141-2154.

Marschner H (1995) Mineral Nutrition of Higher Plants, Ed 2. Academic Press, London

Nussaume L., Kanno S., Javot H., Marin E., Pochon N., Ayadi A., Nakanishi T., and Thibaud M.C. (2011). Phopshate import in plants: focus on the PHT1 transporters. Frontiers in Plant Science 2, 112

Nagy,R., Karandashov,V., Chague,V., Kalinkevich,K., Tamasloukht,M., Xu,G., Jakobsen,I., Levy,A.A., Amrhein,N.,and Bucher,M.(2005). The characterization of novel mycorrhiza-specific phosphate transporters from Lycopersicon esculentum and Solanum tuberosum uncovers functional redundancy in symbiotic phosphate transport in solanaceous species. Plant J. 42, 236-250.

Nagy,R., Vasconcelos,M.J., Zhao,S., Mcelver,J., Bruce,W., Amrhein,N., Raghothama,K.G.,and Bucher,M. (2006). Differential regulation of five Pht1 phosphate transporters from maize (Zea mays L.). PlantBiol. (Stuttg.) 8, 186-197.

Paszkowski U., Kroken S., Roux C. \& Briggs S.P. (2002) Rice phosphate transporters include an evolutionarily divergent gene specifically activated in arbuscular mycorrhizal symbiosis. Proceedings of the National Academy of Sciences, USA 99, 13324-13329

Plassard C. and Dell B. (2010) Phopshorous nutrition of mycorrhizal trees. Tree Physiol. 30, 11291139.

Remans T., Nacry P., Pervent M., Filleur S., Diatloff E., Mounier E., Tillard P., Forde B.G., Gojon A. (2006) The Arabidopsis NRT1.1 transporter participates in the signaling pathway triggering root colonization of nitrate-rich patches. Proceedings of the National Academy of Sciences, USA 103, 19206-19211. 
Rogato A., D’Apuzzo E., Chiurazzi M. (2010) The multiple plant response to high ammonium conditions. The Lotus japonicus AMT1; 3 protein acts as a putative transceptor. Plant Signaling \& Behavior 12, 1594-1596

Rogers C., Oldroyd G. E. (2014) Synthetic biology approaches to engineering the nitrogen symbiosis in cereals. Journal of Experimental Botany (65) 1939-46

Rubio V, Linhares F, Solano R, Martin AC, Iglesias J, Leyva A, Paz-Ares J. (2001) A conserved MYB transcription factor involved in phosphate starvation signalling both in vascular plants and in unicellular algae. Genes Dev 15, 2122-33

Smith, S.E., Jakobsen, I., Grønlund, M. and Smith, F.A. (2011). Roles of arbuscular mycorrhizas in plant phosphorus (P) nutrition: interactions between pathways of $\mathrm{P}$ uptake in arbuscular mycorrhizal(AM) roots have important implications for understanding and manipulating plant $\mathrm{P}$ acquisition. Plant Physiol. 156, 1050-1057.doi:10.1104/pp.111.174581

Su T., Xu Q., Zhang F.C., Chen Y., Li L.Q., Wu W.H. and Chen Y.F. (2015). WRKY42 modulates phosphate homeostasis through regulating phosphate translocation and acquisition in Arabidopsis. Plant Physiol 167, 1579-1591.

Volpe V., Giovannetti M., Sun X.G., Fiorilli V., Bonfante P. (2016). Phosphate transporters LjPT4 and MtPT4 mediate early root responses to phosphate status in non mycorrhizal roots. Plant Cell and Environ doi: 10.1111/pce.12659

Walder F, Brule D, Koegel S, Wiemken A, Boller T, Courty P-E. Plant phosphorus acquisition in a common mycorrhizal network: regulation of phosphate transporter genes of the Pht1 family in sorghum and flax. New Phytol 2015; 205:1632-1645

Wang E., Yu N., Bano S.A., Liu C., Miller A.J., Cousins D., ..., Schultze M. (2014a) A H+-ATPase that energizes nutrient uptake during mycorrhizal symbioses in rice and Medicago truncatula. The Plant Cell 26, 1818-1830 
Wang H., Xu Q., Kong Y.H., Chen Y., Duan J.Y., Wu W.H., and Chen Y.F. (2014b). Arabidopsis WRKY45 transcription factor activates PHOSPHATE TRANSPORTER1;1 expression in response to phosphate starvation. Plant Physiol 164, 2020-2029.

Watts-Williams S.J., Jakobsen I., Cavagnaro T.R. \& Gronlund M. (2015) Local and distal effects of arbuscular mycorrhizal colonization on direct pathway Pi uptake and root growth in Medicago truncatula. Journal of Experimental Botany 66, 4061-4073.

Xie X., Huang W., Liu F., Tang N., Liu Y., Lin H. \& Zhao B. (2013) Functional analysis of the novel mycorrhiza-specific phosphate transporter AsPT1 and PHT1 family from Astragalus sinicus during the arbuscular mycorrhizal symbiosis. New Phytologist 198, 836-852

Xu,G.H., Chague,V., Melamed-Bessudo,C., Kapulnik,Y., Jain,A., Raghothama,K.G., Levy,A.A., and Silber,A.(2007). Functional characterization of LePT4: a phosphate transporter in tomato with mycorrhiza-enhanced expression. J. Exp.Bot. 58, 2491-2501.

Yang S.Y., Grønlund M., Jakobsen I., Grotemeyer M.S., Rentsch D., Miyao A., ..., Paszkowski U. (2012) Nonredundant regulation of rice arbuscular mycorrhizal symbiosis by two members of the Delaux P. M., Radhakrishnan G. V., Jayaraman D., Cheema J., Malbreil M., Volkening J. D., Sekimoto H., Nishiyama T., Melkonian M., Pokorny L., Rothfels C. J., Winter Sederoff H., Stevenson D. W., Surek B., Zhang Y., Sussman M. R., Dunand C., Morris R. J., Roux C., Wong G. K. S., Oldroyd G. E. D., Ané J. M. (2015) The algal ancestor of land plants was pre-adapted for symbiosis. Proceedings of the National Academy of Sciences of the United States of America Publisher's version: 10.1105/tpc.15.00461 